\title{
A Decade-Bandwidth Low-Noise Mixer RFIC with a Distortion-Canceling Output Amplifier
}

\author{
Ahmed M. El-Gabaly*, Hao Li ${ }^{\dagger}$, and Carlos E. Saavedra ${ }^{\dagger}$ \\ *Formerly with Queen's University and now with Peraso Technologies Inc., Toronto, ON, Canada M5J 2L7 \\ ${ }^{\dagger}$ Department of Electrical and Computer Engineering, Queen's University, Kingston, ON, Canada K7L 3N6
}

\begin{abstract}
This paper presents a 1-10 GHz low-noise downconvert mixer RFIC suitable for wideband receivers. A switched transconductor mixing core is adopted to reduce noise at high frequencies. By adding a series inductor to the RF transconductor, a flat 4-5 dB noise figure (NF) and a high gain of $26.5 \mathrm{~dB}$ can be achieved over a broad bandwidth out to $10 \mathrm{GHz}$. A CMOS output amplifier is also integrated on-chip, employing derivative superposition (DS) for high linearity and an OIP3 of $16.5 \mathrm{dBm}$. The circuit consumes less than $20 \mathrm{~mW}$ of dc power and occupies an active chip area of less than $0.2 \mathrm{~mm}^{2}$.
\end{abstract}

\section{INTRODUCTION}

Several topologies exist for wideband low-noise mixing [1]-[5]. Among the most recent and effective approaches are those based on noise canceling. Noise canceling configurations inherently provide some degree of distortion cancellation as well. Derivative superposition (DS) [6]-[10] has also been utilized in mixers for significant distortion cancellation and a high third-order intercept point (IP3). Nevertheless, the operation frequencies of noise-canceling mixers have been mostly limited to about 5 or $6 \mathrm{GHz}$. This is primarily because the auxiliary amplifiers used for noise canceling start to contribute significant noise themselves at higher frequencies. The auxiliary amplifiers' signal gain can be relatively low at high frequencies due to their limited bandwidth, thus making their own input-referred noise considerable.

This paper presents a low-noise down-convert mixer RFIC in $130 \mathrm{~nm}$ CMOS, operating over a decade of frequency bandwidth from $1 \mathrm{GHz}$ to $10 \mathrm{GHz}$. A switched transconductor topology is employed with series inductive peaking to achieve a 4-5 dB noise figure (NF) as well as a high gain of $26 \mathrm{~dB}$ out to $10 \mathrm{GHz}$. A CMOS output amplifier is also integrated onchip, incorporating a new DS technique to realize a high output IP3 (OIP3) of $16.5 \mathrm{dBm}$. Applying DS at the output of the mixer as opposed to its input allows for optimized bandwidth and NF. The chip consumes less than $20 \mathrm{~mW}$ of dc power and occupies an active area of less than $0.2 \mathrm{~mm}^{2}$.

\section{Circuit Description}

\section{A. The Mixing Core}

A circuit schematic of the proposed mixing circuit is shown in Fig. 1. A switched transconductor topology [11] is employed to provide low noise performance over a wide bandwidth out to $10 \mathrm{GHz}$. It consists of three parts: low-noise RF transconductors $\left(M_{1}-M_{2}, M_{3}-M_{4}\right)$, large-swing LO switches $\left(M_{5}-M_{6}, M_{7}-M_{8}\right)$ and high-impedance IF active loads $\left(M_{9}-M_{10}\right)$. The outputs of the LO buffers are accoupled to the source terminals of the RF transconductors. This separates the dc bias current of the RF transconductors from that of the LO buffers, allowing for optimized noise performance and enhanced drive capability respectively.

The RF differential pairs $M_{1}-M_{2}, M_{3}-M_{4}$ are biased in moderate inversion with a low dc bias current $\left(I_{D S} \approx\right.$ $200 \mu \mathrm{A}$ ) to reduce their $1 / \mathrm{f}$ noise. To compensate for the lower transconductance $\left(g_{m}\right)$ in moderate inversion, and to reduce their noise contribution, the width of $M_{1}-M_{2}, M_{3}-M_{4}$ is made relatively large $(W=80 \mu \mathrm{m})$. However a larger device implies higher gate parasitic capacitances $\left(C_{G S}\right)$ diminishing the increase in gain and deteriorating the NF at high frequencies. To mitigate this effect, inductors $L_{G}$ are connected in series with the gates of $M_{1}-M_{2}, M_{3}-M_{4}$ to absorb their parasitic capacitance and widen the bandwidth. By considering the differential-mode half-circuit of $M_{1}-M_{2}$ and $L_{G}$, where the transistor is modelled by $g_{m}, C_{G S}$ and the drain-source noise current $\overline{i_{N D}^{2}}$, the effective transconductance $\left(G_{M}\right)$ and thus the input-referred noise voltage $\left(\overline{v_{N I}^{2}}\right)$ of $M_{1}-M_{2}$ can be approximated as:

$$
G_{M} \approx \frac{g_{m}}{1-\omega^{2} C_{G S} L_{G}}
$$

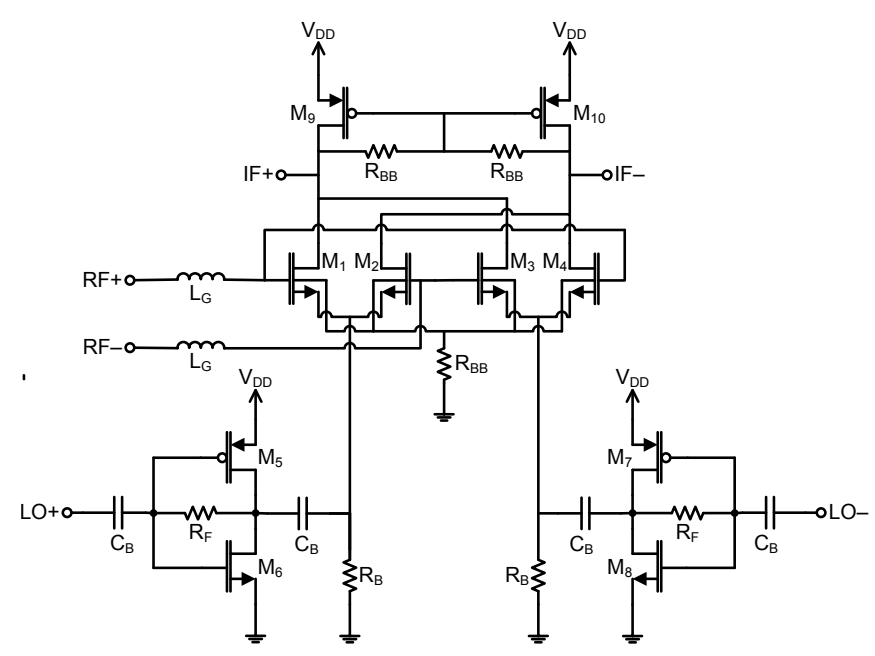

Fig. 1. Circuit schematic of the proposed mixer 


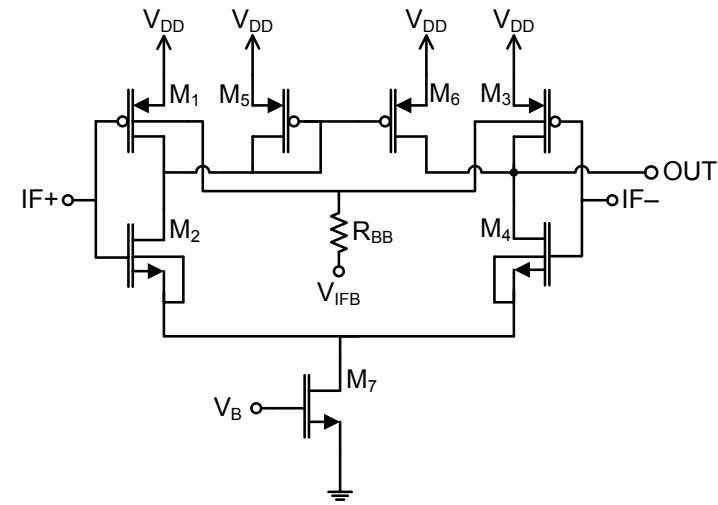

Fig. 2. Circuit schematic of the proposed IF output amplifier

$$
\overline{v_{N I}^{2}} \approx \frac{\left(1-\omega^{2} C_{G S} L_{G}\right)^{2}}{g_{m}^{2}} \overline{i_{N D}^{2}}
$$

Eq. (1) shows that the gain $\left(G_{M}\right)$ of $M_{1}-M_{2}$ can be boosted, while Eq. (2) shows that its NF contribution $\overline{\left(v_{N I}^{2}\right)}$ can be suppressed at high frequencies by using $L_{G}$.

The LO switches $\left(M_{5}-M_{6}, M_{7}-M_{8}\right)$ are comprised of CMOS inverters with shunt-shunt resistive feedback $\left(R_{F}\right)$. Using CMOS inverters provides full rail-to-rail voltage swing, with consistently low output resistance. This enables fast on and off switching of the RF transconductors, reducing unwanted current injection for good gain, noise and linearity performance. The feedback resistor $R_{F}$ provides the dc bias and further lowers the output resistance for high-frequency LO operation. A major advantage of the mixer's switched transconductor topology is that the noise generated by the LO switches (both 1/f and thermal) appears as common-mode noise at the IF outputs, which is rejected in differential mode [11]. This is particularly important at high LO frequencies, mitigating the rise in NF due to the lower switching speed and conversion gain.

\section{B. IF amplifier}

A circuit schematic of the IF output amplifier is shown in Fig. 2. The IF amplifier converts the mixer's differential IF port $(I F+$ and $I F-)$ to a single-ended output and drives the relatively low impedance $50 \Omega$ load. It consists of CMOS transconductors $M_{1}-M_{2}, M_{3}-M_{4}$, a PMOS current mirror $M_{5}-M_{6}$ and an NMOS current source $M_{7}$. The PMOS current mirror $M_{5}-M_{6}$ mirrors the current from one differential side (or half) to the other, performing the differential to singleended conversion.

The design of the IF amplifier is optimized for linearity, since the mixing core has sufficient gain to make the amplifier's noise contribution negligible. Applying DS in the IF amplifier and not in the mixing core reduces its effect on the RF bandwidth and NF. In comparison with common-source NMOS transconductors, the CMOS transconductors $M_{1}-M_{2}$, $M_{3}-M_{4}$ offer larger voltage swings and better linearity at the cost of reduced frequency bandwidth. The drain-source current $i_{D S}$ of the NMOS and PMOS devices can be described using the well-known power series:

$$
i_{D S}=I_{D S}+g_{m 1} v_{G S}+g_{m 2} v_{G S}^{2}+g_{m 3} v_{G S}^{3}+\ldots
$$

where $I_{D S}$ is the dc bias drain-source current, $v_{G S}$ is the gatesource voltage and $g_{m n}$ are the transconductance coefficients. The higher-order terms in the series, $v_{G S}^{2}$ and $v_{G S}^{3}$, describe the second and third-order non-linear distortion. The secondorder transconductance $g_{m 2}$ of the PMOS devices $M_{1}, M_{3}$ has an opposite polarity to that of the NMOS devices $M_{2}, M_{4}$, thus the second-order non-linear currents are suppressed upon summation at the output. Feedback of second-order non-linear currents through parasitic capacitances can otherwise give rise to third-order distortion after subsequent second-order mixing with the fundamental tones [6].

Furthermore, to lessen the amount of third-order distortion produced by $g_{m 3} v_{G S}^{3}$ in (3), the body terminals of the PMOS devices $M_{1}$ and $M_{3}$ are biased at $V_{I F B}=2.4 \mathrm{~V}>V_{D D}$. The desired goal is to increase the body-source voltage $V_{B S}$ and thus the threshold voltage $V_{T}$ of $M_{1}$ and $M_{3}$, which decreases their dc current $I_{D S}$ for weaker channel inversion. This changes the sign of the third-order transconductance $g_{m 3}$ of $M_{1}$ and $M_{3}$ from negative to positive, which is now opposite to that of the NMOS devices $M_{2}$ and $M_{4}$ (biased in strong inversion). Therefore the third-order distortion currents can be rejected upon summation at the outputs.

\section{Simulation and Measurement Results}

The mixer was fabricated in a standard $0.13 \mu \mathrm{m}$ CMOS process and a photograph of the IC is shown in Fig. 3. It occupies a die area of $1 \mathrm{~mm}^{2}$ including bonding pads, while the core circuit area is $405 \times 480 \mu \mathrm{m}^{2}$. The circuit consumes less than $20 \mathrm{~mW}$ of dc power from a $1.5 \mathrm{~V}$ supply.

The broadband mixer IC was measured directly on-wafer using $40 \mathrm{GHz}$ coplanar waveguide (CPW) probes. A $50 \mathrm{GHz}$ spectrum analyzer with a noise source was used for gain, NF and linearity measurements. For all measurements, the LO power was fixed at $-4 \mathrm{dBm}$, and the $\mathrm{RF}\left(f_{R F}\right), \mathrm{LO}\left(f_{L O}\right)$ and IF $\left(f_{I F}\right)$ frequencies were chosen such that $f_{R F}=f_{L O}+f_{I F}$.

Fig. 4 shows the measured gain and DSB NF of the mixer versus LO frequency in comparison with simulation results. In these measurements, the IF frequency was fixed at $250 \mathrm{MHz}$. It is clear that a high gain of $26.5 \pm 1.0 \mathrm{~dB}$ and a low NF of

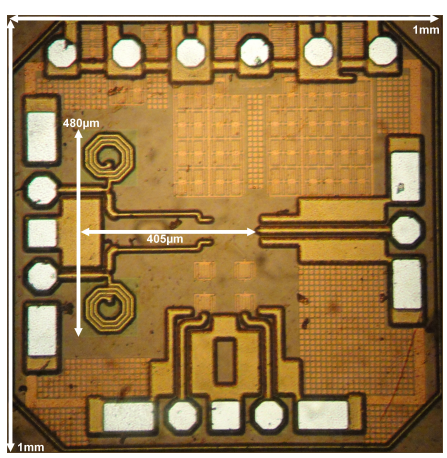

Fig. 3. Photograph of Broadband Mixer IC. 


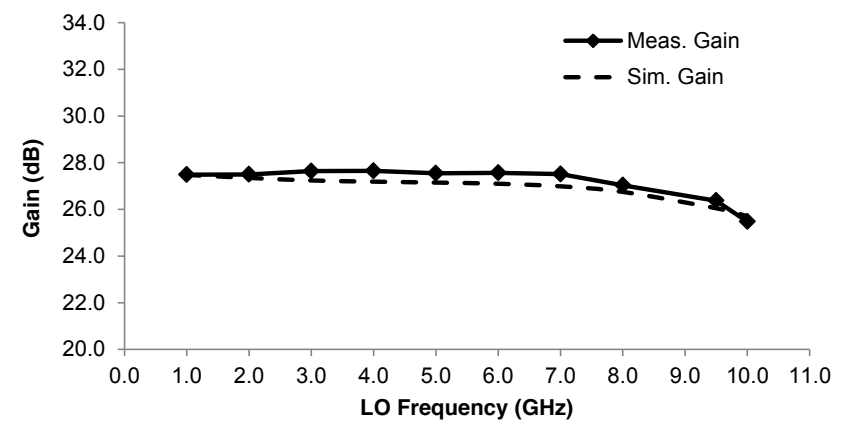

(a)

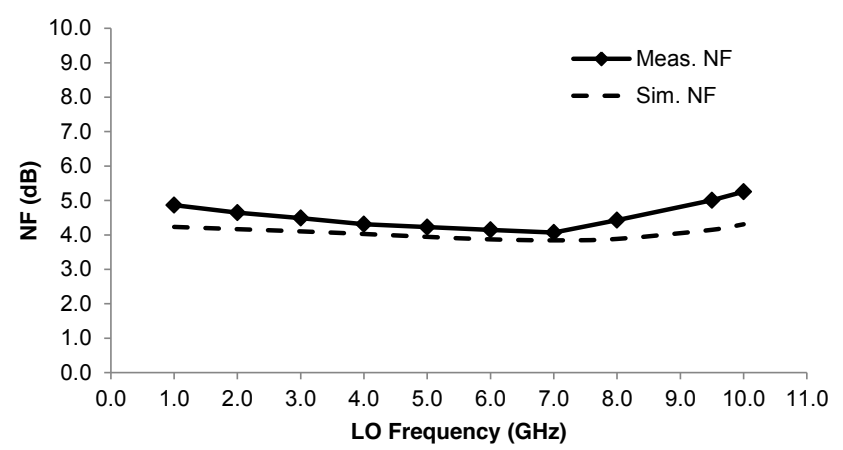

(b)

Fig. 4. Mixer performance over LO frequency: (a) gain and (b) NF.

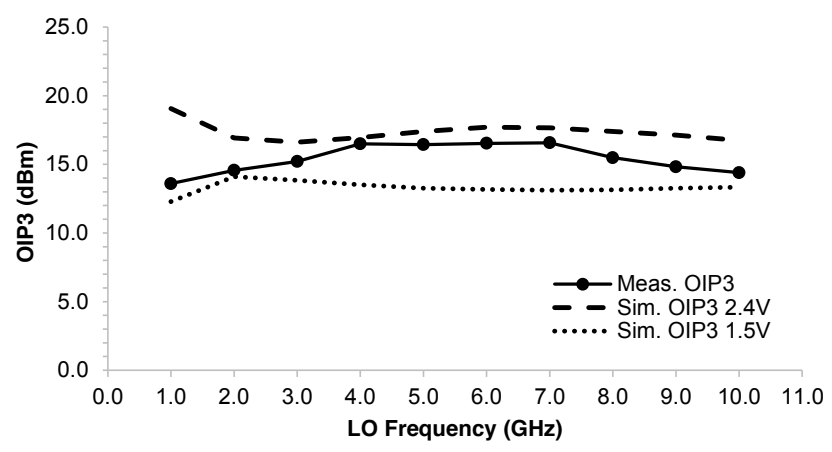

Fig. 5. Measured mixer OIP3 performance.

$4.6 \pm 0.6 \mathrm{~dB}$ are achieved from $1 \mathrm{GHz}$ to $10 \mathrm{GHz}$. The NF is as low as $4.0 \mathrm{~dB}$ at $7 \mathrm{GHz}$. These results demonstrate that the mixer's 3-dB bandwidth exceeds $10 \mathrm{GHz}$. The DSB NF was also verified over a broad range of IF frequencies from $5 \mathrm{MHz}$ to $500 \mathrm{MHz}$. The $\mathrm{NF}$ is below $6 \mathrm{~dB}$ down to an IF frequency of $5 \mathrm{MHz}$, indicating low 1/f noise.

The OIP3 and output $1-\mathrm{dB}$ compression (OP1dB) points were characterized from $1 \mathrm{GHz}$ to $10 \mathrm{GHz}$. For these measurements, the IF frequency was fixed at $200 \mathrm{MHz}$. The frequency spacing between the two tones for the OIP3 measurement was $10 \mathrm{MHz}$. Fig. 5 is a plot of the measured and simulated OIP3 versus LO frequency. The OIP3 peaks to $16.5 \mathrm{dBm}$ at the center of the band, but drops to $14.4 \mathrm{dBm}$ towards the edges. The simulated OIP3 without distortion cancellation (i.e. $V_{I F B}=1.5 \mathrm{~V}$ in the IF amplifier), is also shown in Fig. 5. Meanwhile, the mixer's OP1dB (not shown) ranged between
TABLE I

SUMMARY OF THE MIXER'S PERFORMANCE

\begin{tabular}{ccccc}
\hline Characteristic & This work & {$[3]$} & {$[8]$} & {$[5]$} \\
\hline Chip Area $\left(\mathrm{mm}^{2}\right)$ & $\mathbf{0 . 2}$ & 0.315 & 0.10 & 1.21 \\
DC Power (mW) & $\mathbf{2 0}$ & 34.5 & 20 & 25.5 \\
Gain (dB) & $\mathbf{2 6 . 5}$ & 17.5 & 11 & 15 \\
Bandwidth (GHz) & $\mathbf{1 - 1 0}$ & $1-5.5$ & 1 & $0.5-5.8$ \\
DSB NF (dB) & $\mathbf{4 . 6} \pm \mathbf{0 . 6}$ & 3.9 & 15.9 & 4.2 \\
OIP3 & $\mathbf{1 6 . 5}$ & 15.6 & 17.5 & - \\
\hline
\end{tabular}

$+1 \mathrm{dBm}$ and $+3 \mathrm{dBm}$ over the band. Table I summarizes the proposed mixer's performance.

\section{CONCLUSION}

A 1-10 GHz down-convert mixer with an integrated output amplifier has been developed in $130 \mathrm{~nm}$ CMOS. The switchedtransconductor configuration is adopted with series inductive peaking for high gain $(26.5 \mathrm{~dB})$ and low $\mathrm{NF}(4-5 \mathrm{~dB})$ up to $10 \mathrm{GHz}$. DS is effectively incorporated in the output amplifier using the body bias of the PMOS devices, to achieve a high OIP3 of $16.5 \mathrm{dBm}$. The circuit consumes less than $20 \mathrm{~mW}$ of dc power and occupies an active chip area below $0.2 \mathrm{~mm}^{2}$.

\section{REFERENCES}

[1] A. Amer, E. Hegazi, and H. Ragaie, "A 90-nm wideband merged CMOS LNA and mixer exploiting noise cancellation," IEEE J. Solid-State Circuits, vol. 42, no. 2, pp. 323-328, Feb 2007.

[2] S. Blaakmeer, E. Klumperink, D. Leenaerts, and B. Nauta, "The blixer, a wideband balun-LNA-I/Q-mixer topology,' IEEE J. Solid-State Circuits, vol. 43, no. 12 , pp. 2706-2715, Dec 2008.

[3] S. Ho and C. Saavedra, "A CMOS broadband low-noise mixer with noise cancellation," IEEE Trans. Microw. Theory Techn., vol. 58, no. 5, pp. 1126-1132, May 2010.

[4] D. Na and T. W. Kim, "A 1.2 V, 0.87-3.7 GHz wideband low-noise mixer using a current mirror for multiband application," IEEE Microw. Compon. Lett., vol. 22, no. 2, pp. 91-93, Feb 2012.

[5] B. Guo, H. Wang, and G. Yang, "A wideband merged CMOS active mixer exploiting noise cancellation and linearity enhancement," IEEE Trans. Microw. Theory Techn., vol. 62, no. 9, pp. 2084-2091, Sept 2014.

[6] T. W. Kim, B. Kim, and K. Lee, "Highly linear receiver front-end adopting MOSFET transconductance linearization by multiple gated transistors," IEEE J. Solid-State Circuits, vol. 39, no. 1, pp. 223-229, Jan 2004.

[7] J. Lee, J. Lee, B. Kim, B.-E. Kim, and C. Nguyen, "A highly linear lownoise amplifier using a wideband linearization technique with tunable multiple gated transistors," in IEEE Radio Freq. Integr. Circuits Symp., June 2013, pp. 181-184.

[8] M. Wang, S. He, and C. E. Saavedra, "+14 dB Improvement in the IIP $_{3}$ of a CMOS Active Mixer through Distortion Cancellation," in IEEE Int Wireless Symposium, April 2013, pp. 1-4.

[9] A. El-Gabaly, D. Stewart, and C. Saavedra, "2-W broadband GaN poweramplifier RFIC using the $f_{T}$ doubling technique and digitally assisted distortion cancellation," IEEE Trans. Microw. Theory Techn., vol. 61, no. 1, pp. 525-532, Jan 2013.

[10] K.-H. Liang, C.-H. Lin, H.-Y. Chang, and Y.-J. Chan, "A new linearization technique for CMOS RF mixer using third-order transconductance cancellation," IEEE Microw. Compon. Lett., vol. 18, no. 5, pp. 350-352, May 2008.

[11] E. Klumperink, S. Louwsma, G. Wienk, and B. Nauta, "A CMOS switched transconductor mixer," IEEE J. Solid-State Circuits, vol. 39 no. 8, pp. 1231-1240, Aug 2004. 